\title{
This Month in AJP
}

\section{Signaling through the Bob Receptor as a Possible Cause for HIV Enteropathy}

HIV-infected individuals frequently develop an enteropathy consisting of malabsorption and increased small intestine permeability with diarrhea in the absence of enteric infection. The major HIV co-receptors CCR5 and CXCR4 are present in enteric epithelium but are located at the luminal surface. Clayton et al (Am J Pathol 2001, 159:1933-1939) hypothesized that receptors present on the basolateral surface of intestinal cells would be more plausible mediators of HIV effects. They show that the minor HIV co-receptor GPR 15/Bob is abundantly expressed on the basolateral surface and mediates calcium signaling by the HIV-1 envelope surface protein gp120. The data suggest that gp210 120-induced Bob activation is a likely cause of HIV enteropathy.

\section{Presenilin 1 Binding Protein May Be Involved in Neurodegeneration in Alzheimer's Disease}

Familial forms of Alzheimer's disease (AD) are associated with mutations in the amyloid precursor protein and presenilins (PS) 1 and 2. Presenilins can interact with multiple proteins but their biological role is unclear. A novel presenilin 1 binding protein (PBP) was recently identified. PBP co-immunoprecipitates with PS1 in hippocampus cell lysates and binds to PS1 in the yeast two-hybrid system. Chen et al (Am J Pathol 2001, 159:1597-1602) report that PBP increases tau phosphorylation and is closely associated with neurofibrillary tangles in brains of AD patients. Chen et al suggest that PBP might have a role in the generation of neurofibrillary tangles through its effect on tau phosphorylation.

\section{Platelet-Derived Growth Factor B-Chain Is Not Required for Wound Healing}

Given the biological effects of platelet-derived growth factor (PDGF) in cultured cells, it is logical to assume that wound repair is enhanced by the release of PDGF from platelets and macrophages at wound sites. Buetow et al (Am J Pathol 2001, 159:1869-1876) tested this hypothesis by establishing chimeric mice in which the hemopoietic system of a normal adult mouse was replaced by the hemopoietic system of a PDGF B-chain null mouse (-/-) or that of a B-chain positive animal $(+/+)$ as a control. They initiated local granulation tissue formation by foreign body reaction or as a response to thrombosis. In both models, absence of PDGF B-chain did not decrease the extent of granulation tissue formation and the degree of vascularization of the lesions was greatly increased. Buetow et al speculate that PDGFB expression may inhibit the recruitment of pericytes and smooth muscle cells required for neovascularization.

\section{Myopodin Loss May Be a Marker for Invasive Prostate Cancer}

A large number of studies have been published on the expression of specific genes which may serve as prognostic markers in prostate cancer. Yet, not much is known about genes which may be critical for the development of invasive prostate cancers. Lin et al (Am J Pathol 2001, 159:1603-1612) performed a genome-wide search for sequences which are deleted in invasive cancers using the "differential subtraction chain" technique. They identified a region in chromosome 4 q25 containing the gene for myopodin that was partially or completely deleted in $50 \%$ of prostate cancers and $80 \%$ of invasive prostate cancers. Myopodin shows homology with synaptopodin, a protein associated with podocyte and neuron differentiation. Although a clear function for prostatic myopodin has not been established, the loss of the protein is highly correlated with invasiveness and might serve as a reliable prognostic marker.

\section{Pancreatoblastomas Have Similar Molecular Defects as Hepatoblastomas but Differ from Pancreatic Adenocarcinomas}

Pancreatoblastomas are malignant neoplasms of the pancreas occurring in children and occasionally in adults. The molecular pathogenesis of these tumors is unknown. Abraham et al (Am J Pathol 2001, 159:1619-1627) hypothesized that pancreatoblastomas may have genetic similarities to other infantile embryonic tumors such as hepatoblastomas. They analyzed a series of pancreatoblastomas for changes found in hepatoblastomas (mutations in APC/ $\beta$-catenin, $5 \mathrm{q}$, and $11 \mathrm{p}$ allelic losses) as well as mutations in K-ras, p53, and DPC4 changes commonly found in adult pancreatic adenocarcinomas. Allelic loss of chromosome $11 \mathrm{p}$ was present in six of seven informative cases and alterations in APC/ $\beta$ catenin were detected in six of nine cases. In contrast, K-ras and p53 alterations were not found and loss of DPC4 protein expression was present in two of nine cases. The results established that pancreatoblastomas are genetically distinct 
from pancreatic ductal adenocarcinomas and closely resemble hepatoblastomas in their molecular pathogenesis. Abraham et al also suggest that pancreatoblastomas may be an extracolonic manifestation of familial adenomatous polyposis (FAP) and present a case of an adult FAP patient with pancreatoblastoma.

\section{Microarray Analyses Using Frozen Tissues}

Tissue microarray technology involves analyses of up to 1000 cylindrical tissue cores arrayed on a recipient block. Serial sections of the block can be made to analyze DNA, RNA, and proteins on a glass slide. The technique permits the rapid analysis of multiple tissue samples and facilitates the correlation between morphological features and molecular alterations in individual tumors. The original technology involved obtaining tissue cores from paraffin-embedded tissue blocks which are then deposited into a single paraffin block. Although this technique has been successfully used, it presents problems related to the preservation of antigens and RNA degradation in fixed-paraffin embedded tissue. Fejzo and Slamon (Am J Pathol 2001, 159:1645-1650) describe a modification of tissue array technology using frozen tissues embedded in OCT and arrayed into an OCT block. The frozen tissue arrays offer significant advantages over the original techniques and can be used for RNA, DNA, and protein analyses. 